\title{
Qualität statt Partizipation und Gleichheit? Eine Bemerkung zum epistemischen Sinn von Demokratie ${ }^{1}$
}

Theorien deliberativer Demokratie ${ }^{2}$ finden zunehmend im Kontext der Debatte um die Legitimität der Europäischen Union sowie transnationaler Politik Verwendung. In der Suche nach möglichen Arrangements legitimen transnationalen Regierens sind sie zum Anker geworden, auf den sich längst nicht mehr nur Autoren beziehen, die der Tradition der Kritischen Theorie nahestehen. Die Konsequenz dieser Entwicklung ist paradox. Neben dem Vorwurf, Theorien deliberativer Demokratie seien realitätsfern, werden sie nun im selben Atemzug als zu affirmativ dargestellt: Sie seien » überangepasst und realitätsentrückt « ${ }^{3}$ - so lautet der Vorwurf.

Dieser Einwand führt aber ins Leere, weil dabei eine Ambivalenz übersehen wird: Er unterscheidet nicht zwischen der anwendungsorientierten Debatte über legitimes Regieren jenseits des Nationalstaats, in der der Begriff Deliberation selektiv für bestimmte Zwecke herausgefiltert wird, und der Rolle, die der Begriff Deliberation im Kontext seines demokratietheoretischen Entstehungszusammenhangs spielt. Während im ersten Fall der Deliberation für sich genommen ein legitimierender Mehrwert unterstellt und sie dadurch regelmäßig überbewertet wird, hängt im zweiten Fall die normative Bedeutung von Deliberation von ihrer engen Verzahnung mit anderen Aspekten demokratischer Ordnung ab. Übersehen wird, kurz gesagt, dass Deliberation in der demokratietheoretischen Herleitung jene Prozesseigenschaft bezeichnet, die bereits im Modell nationalstaatlicher repräsentativer Demokratie die in den politischen Prozess eingespeisten individuellen Interessen erst in

1 Ich danke Reinhard Blomert, Rainer Schmalz-Bruns, Oliver Schmidtke sowie den anonymen Gutachtern für wertvolle Kritik und Kommentare. Dem Käte Hamburger Kolleg »Politische Kulturen der Weltgesellschaft « der Universität Duisburg-Essen danke ich für die größzügige Unterstützung bei der Fertigstellung dieses Artikels.

2 Bessettes (1980) These, dass die Gründungsväter mit der US-amerikanischen Verfassung die Einrichtung einer deliberativen Demokratie beabsichtigten, hat den Anstoß zu einer mittlerweile weit ausgreifenden politikwissenschaftlichen Diskussion gegeben. Neben der Auseinandersetzung darüber, welche Rolle Deliberation, also Prozesse der Beratung und Diskussion, für die Erzeugung demokratischer Legitimität spielen (vgl. Manin 1987; Cohen 1989; Knight, Johnson 1994; Gutmann, Thomson 1996; Bohman, Rehg 1997; Elster 1998; Passerin D'Entreves 2002; Besson, Martí 2006), finden sich in jüngerer Zeit vermehrt empirisch orientierte Studien, die die Prämissen des Modells deliberativer Demokratie und Realisierungsbedingungen von Deliberation in der Politik untersuchen (vgl. Bächtiger, Steiner 2005; Chambers 2003; Thompson 2008; Schaal, Ritzi 2009).

3 Höreth 2009. 
kollektiv verbindliche Entscheidungen übersetzt, die als legitim akzeptiert werden können.

Dieser Zusammenhang soll anhand einer prominenten Variante der Theorie deliberativer Demokratie, der Diskurstheorie des demokratischen Rechtsstaats von Jürgen Habermas, ${ }^{4}$ erläutert werden. Habermas' Theorie eignet sich deshalb, weil sie vielen als Ausdruck der Vorstellung von »Deliberation ohne Demokratie « erscheint, die zunehmend als eigenständiger Weg zur Legitimierung transnationaler Politik nahegelegt wird. Gegen diese Interpretation spricht, so werde ich im Folgenden argumentieren, dass aus der Perspektive der Habermas'schen Diskurstheorie politische Entscheidungsfindung via Deliberation ohne Demokratie kein tolerierbarer Zustand, sondern schlichtweg illegitim ist. Der Knackpunkt in der Auseinandersetzung ist die Annahme eines epistemischen Sinns, den Habermas zufolge der demokratischen Prozess hat und der für die Legitimität politischer Ordnung - gleich ob nationaler, trans- oder supranationaler Gestalt - entscheidend ist. Mit dem epistemischen Sinn von Demokratie ist gemeint, dass Bürger vom demokratischen Prozess erwarten, Entscheidungen hervorzubringen, die nicht nur an ihren Interessen ausgerichtet sind, sondern darüber hinaus »vernünftig «, das heißt der Sache angemessen und gerecht sind. Für Vertreter eines voluntaristischen Verständnisses von Demokratie dagegen verbindet sich mit der Annahme eines epistemischen Sinns von Demokratie von vornherein ein elitäres Demokratieverständnis, in dem die demokratischen Ideale von Partizipation und politischer Gleichheit zum Zwecke der Erzeugung vernünftiger Ergebnisse aufgegeben werden. ${ }^{5}$

Um die Verbindung von Deliberation und Demokratie herauszustreichen, werde ich nachfolgend Habermas' These eines epistemischen Sinns von Demokratie gegen drei zentrale Einwände verteidigen, die bezüglich der Rolle von Partizipation, Gleichheit und Institutionen im Modell deliberativer Demokratie ${ }^{6}$ erhoben worden sind. Dabei geht es nicht darum, die Einwände pauschal zurückzuweisen. Vielmehr soll gezeigt werden, dass alle drei Einwände jeweils wichtige Aspekte der Demokratie betonen, die jedoch erst über ihre Integration in einem deliberativen Modell zu einem unverkürzten Verständnis der normativen Bedeutung des demokratischen Prozesses führen.

Dem ersten Einwand zufolge führe die Theorie deliberativer Demokratie zu einer Rationalisierung der Demokratietheorie, weil sie die Bedeutung von Partizipation

\section{Habermas 1992.}

5 Vereinfachend gesagt, unterscheidet sich ein voluntaristisches von einem epistemischen Verständnis von Demokratie durch die Annahme, dass »a key characteristic of a democracy is the continuing responsiveness of the government to the preferences of the citizens « (Dahl 1971, S. 1). Während ersteres die demokratische Legitimität von Entscheidungen über deren Nähe zu den Willensäußerungen der Bürger erklärt, verweist letzteres auf einen kognitiven Gehalt der Entscheidungen, welcher auf dem Wege der Verarbeitung von in den politischen Prozess eingespeisten Willensäußerungen (inputs) zu Entscheidungen (outputs) erzeugt wird. Ich komme darauf später zurück.

6 Wenn ich fortan vereinfachend von Modell oder Theorie deliberativer Demokratie spreche, beziehe ich mich damit, sofern nicht anders gekennzeichnet, auf Habermas' Diskurstheorie des Rechts und des demokratischen Rechtsstaats. 
dadurch unterminiere, dass sie demokratische Legitimität mit Bezug auf die Qualität demokratischer Entscheidungen begründet. Dagegen argumentiere ich, dass die Annahme eines epistemischen Sinns von Demokratie noch nichts über die Bedeutung aussagt, die Bürgerbeteiligung in der Begründung der Rationalität von Entscheidungen einnimmt (Kapitel 1). Zweitens ist der Vorwurf vorgebracht worden, die von der deliberativen Demokratietheorie angenommene Qualität demokratischer Entscheidungen lasse sich nur dadurch erzielen, dass das Ideal politischer Gleichheit verletzt werde. Dem entgegen soll gezeigt werden, dass Deliberation im demokratischen Prozess eine wesentliche Voraussetzung zur Herstellung politischer Gleichheit ist (Kapitel 2). Schließlich wende ich mich gegen die Annahme, die institutionentheoretischen Betrachtungen der deliberativen Demokratietheorie konzentrierten sich auf die Rolle von (Experten-)Deliberation und blendeten demokratische Mechanismen wie parlamentarische Repräsentation und freie und allgemeine Wahlen aus. Aus der Perspektive der Theorie deliberativer Demokratie hängt die Legitimität (auch) transnationalen Regierens davon ab, dass Deliberationsprozesse in eine Gesamtarchitektur politischer Organisation eingebettet sind, in der eben jene demokratischen Mechanismen wirksam institutionalisiert sind - wenngleich das unter veränderten, transnationalen Vorzeichen zu geschehen hat (Kapitel 3).

\section{Qualität statt Partizipation?}

Hubertus Buchstein und Dirk Jörke zufolge vollzieht sich im gegenwärtigen politikwissenschaftlichen Diskurs eine semantische Transformation des Demokratiebegriffs, die in ihrer Bedeutung mit dem Übergang vom antiken zum neuzeitlichen Demokratiebegriff vergleichbar sei. Demnach sei der antike Demokratiebegriff ein "Negativbegriff « insofern, als seine Überlieferung auf Quellen basiere (Platon, Aristoteles, Cicero, Polybios), die durchweg entschiedene Kritik an der demokratischen Regierungsform übten. Während die Demokratie dort als eine Regierungsform erscheine, in der die Beteiligung unqualifizierter Bürger Entscheidungsgänge unnötig kompliziere, hat sich nach Buchstein und Jörke im Zuge der amerikanischen und französischen Revolution sowie der sich anschließenden Ausbreitung des Wahlrechts im 19. Jahrhundert Demokratie zu einem positiv besetzten Begriff gewandelt. Damit verband sich, so die Autoren weiter, ein Wandel der Institutionenordnung demokratischen Regierens. Im Unterschied zum antiken Begriff Demokratie nun nicht mehr nach dem Modell athenischer Demokratie verstanden, in der die Versammlung der Bürger direkt und ohne institutionelle Einschränkungen Entscheidungen trifft. Stattdessen werde im modernen Demokratiebegriff der verfassungsmäßig garantierte Vorrang individueller Abwehrrechte gegen Mehrheitbeschlüsse sowie eine repräsentative Organisation demokratischen Entscheidens vorausgesetzt. Die starke Stellung der Bürgerbeteiligung habe durch die Aufnahme der Prinzipien rechtsstaatlicher, gewaltenteiliger und repräsentativer Organisation in den Demokratiebegriff eine deutliche Abschwächung erfahren. Dass der Begriff Demokratie diese weitreichenden Veränderungen überlebte, führen Buchstein und Jörke auf die fortdauernde Attraktion zurück, die von seinen beiden Wortbestandteilen ausgeht: 
»Die rhetorische Bezugnahme auf das Volk und die Herrschaft stellt einen - wie auch immer abgeschwächten - Bezug zu partizipativen Komponenten in politischen Systemen her und versieht sie mit breitenwirksamer Legitimation « ${ }^{7}$

Genau dieses Verhältnis zwischen Volk, Herrschaft und Partizipation sehen Buchstein und Jörke im gegenwärtigen politikwissenschaftlichen Diskurs in Auflösung begriffen. Vormals Bedeutungskern, der seiner historischen Durchsetzungskraft zugrunde lag, sei »die partizipative Komponente des Demokratiebegriffs jedoch zu einem Ballast geworden, der seinem weiteren Erfolg im Wege steht $«{ }^{8}$ Komplexitätszunahme, Pluralisierung und Globalisierung haben demnach das "akademische Demokratietheorisieren « einem Druck ausgesetzt, auf den dieses mit einem Rationalisierungsschub reagiere - allen voran die Theorie deliberativer Demokratie als »avancierteste Version des Rationalitätsparadigmas « ${ }^{9}$ Demnach tilge die deliberative Demokratietheorie sukzessive die (in transnationalen Kontexten immer schwerer zu realisierende) Idee der Partizipation aus dem Bedeutungskern von Demokratie. Im Mittelpunkt ihrer Aufmerksamkeit stehe nach Buchstein und Jörke nunmehr die Frage, wie die Qualität von Entscheidungen bewertet und erhöht werden kann: »Demokratietheorie ist heute output-orientiert « und rechtfertige Demokratie als politisches System, »das gute im Sinne von rationalen< Politikergebnissen erzeugt $« .{ }^{10}$ Dem stehe Partizipation eher im Wege. Entsprechend werde Partizipation nur dann als relevant gesehen, wenn sie zur Steigerung der Vernünftigkeit von Entscheidungen beitragen könne. Indem aber politische Beteiligung nicht mehr als Ziel, sondern nur noch als eines von vielen Mitteln zum Zweck rationaler Ergebnisse verstanden werde, so Buchstein und Jörke weiter, begebe sich die deliberative Demokratietheorie in einen Gegensatz zum Alltagsverständnis von Demokratie. »Der vollständigen Unterordnung der demokratischen Beteiligung unter die Rationalitätszumutungen moderner Politik « stehe die alltägliche Verwendungsweise des Demokratiebegriffs entgegen, mit der nach wie vor das »Versprechen auf wirksame Beteiligung 'von unten « aufgerufen werde. ${ }^{11} \mathrm{Um}$ der schleichenden Tendenz zur Entdemokratisierung, die sich mit der Ausbreitung des Rationalitätsparadigmas ergebe, wirksam entgegenzuarbeiten - so der kritische Schluss der Autoren -, bedürfe es einer Betonung des ursprünglichen Bedeutungskerns von Demokratie. Es gelte demnach, »die für das bisherige Überleben des Demokratiebegriffs verantwortliche Versprechenskomponente explizit in das Zentrum der Demokratietheorie zu stellen « 12 - die Orientierung der deliberativen Demokratietheorie auf die Qualität der Entscheidungen des demokratischen Prozesses müsse umgekehrt werden in eine Orientierung, die Demokratie als Regierungsform der politischen Beteiligung diskutiert.

7 Buchstein, Jörke 2003, S. 474.

8 Ebd.

9 Ebd., S. 491; vgl. auch Buchstein 2009; Jörke 2009.

10 Buchstein, Jörke 2003, S. 475.

11 Ebd., S. 476, 482.

12 Ebd., S. 490; Hervorhebung im Original. 
Buchsteins und Jörkes Erinnerung an die Rolle des Demokratiebegriffs im politischen Alltagsgebrauch ist wichtig. Gegen alle Entstellungen, die der Demokratiebegriff mitunter im theoretischen Gebrauch wie auch in der politischen Praxis der Herrschaftslegitimation erfährt, wird durch sein Aufrufen ein Versprechen aktualisiert, das eine ungebrochene Emanzipationskraft in sich birgt. Es ist aber fraglich, ob dieses Versprechen zutreffend als ein Versprechen auf bloße Partizipation begriffen werden kann. Ein alternativer Blick auf die semantische Transformation der Bedeutung des Demokratiebegriffs im Übergang zur Moderne stützt eine andere Lesart. Ideengeschichtlich betrachtet, hält, wie John Dunn argumentiert, das emanzipatorische Potential, das sich mit dem Versprechen der Demokratie heute verbindet, erst im Verlauf beziehungsweise nach der amerikanischen und französischen Revolution Einzug in das Alltagsverständnis. ${ }^{13}$ Zuvor verband sich Dunn zufolge mit Demokratie fast 2.000 Jahre lang ein Verständnis von Bürgerbeteiligung, das politische Denker nicht als wünschenswert, sondern als den Bedingungen des Gemeinwohls entgegenstehend wahrnahmen. Folgt man Pierre Rosanvallon, lässt sich diese negative Konnotation von Demokratie auf die Wirkung einer »Einstimmigkeitskultur « im politischen Denken zurückführen, welche bis in die Neuzeit vorherrschte. ${ }^{14}$ Rosanvallon zufolge definierte sich das Gemeinwohl in der römischen Gesellschaft wie auch in germanischen und gallischen Stammeskulturen sowie in der Kirche der ersten Jahrhunderte bis hin zu den italienischen Stadtrepubliken des 12. Jahrhunderts "nicht durch ein Zusammentreffen von Standpunkten, es ist als ein moralisch-sozialer Tatbestand unmittelbar geboten $"{ }^{15}$ Frühe Formen der Partizipation beziehungsweise Wahlen sind demnach nicht im Sinne demokratischer Beteiligung, also als Möglichkeit zur individuellen Meinungsäußerung und Widerspruch zu begreifen, sondern als Akte der Akklamation, die Einvernehmen zeigen und die Einheit und den guten Zustand der Gemeinschaft bekräftigen. Vor diesem Hintergrund betrachtet, ist die semantische Transformation des Demokratiebegriffs in der Moderne mehr als eine bloße Abschwächung der partizipativen Komponente zum Zwecke ihrer Umsetzbarkeit in größeren Gesellschaften, denn: Warum sollte Partizipation im demokratischen Sinn überhaupt wünschenswert sein?

Es macht dagegen mehr Sinn, die semantische Transformation des Demokratiebegriffs im Kontext eines Bedeutungswandels des Begriffs der politischen Partizipation zu betrachten, der mit einer Ablösung der »Einstimmigkeitskultur « in Verbindung steht. Die Transformation des Demokratiebegriffs im 18. Jahrhundert hängt dann mit dem Umstand zusammen, dass sich seither ein gesellschaftliches Bewusstsein der Individualität etabliert hat, welches Hand in Hand mit der Ausbildung des Gerechtigkeitsideals gleicher individueller Freiheit geht. Damit haben sich die Koordinaten in der Konstellation politischen Denkens grundlegend verschoben. Die neue gesellschaftliche Rolle des Individuums tritt in Spannung zum Ideal der Einheit der Gemeinschaft, welches seinerseits in einem gewandelten Verständnis von

13 Dunn 2005.

14 Rosanvallon 2010, S. $27 \mathrm{ff}$.

15 Ebd., S. 30. 
Staat aufgeht. ${ }^{16}$ Staat und Individuum bilden fortan zwei Pole, die sich aber wechselseitig bedingen und so erst gemeinsam den Raum der Gesellschaft aufspannen. Larry Siedentop zufolge bildet die Doktrin staatlicher Souveränität eine praktische Voraussetzung für die gesellschaftsweite Ausbildung der sozialen Rolle des Individuums und des Gedankens gleicher individueller Freiheit. »Der Staat ist per Definition gebunden an den Wert der Gleichheit in mindestens dieser Hinsicht: er bedeutet die gleiche Unterwerfung unter eine höchste Autorität oder Macht, den Souverän. [...] Das verleiht dem Staat, de facto, eine fundamentale Rolle im Sozialisationsprozess. " ${ }^{17}$ Demokratietheoretisch gesehen, ist es aus zwei Gründen wichtig, sich diesen Zusammenhang vor Augen zu führen. Zum einen lässt sich so der volle Umfang des Versprechens der Demokratie im Alltagsverständnis besser nachvollziehen, der auch eine epistemische Funktion des demokratischen Prozesses einschließt. Zum anderen, und darauf gehe ich später ein (unter Kapitel 3), lassen sich vor diesem Hintergrund die Schwierigkeiten deutlicher benennen, die sich für eine Demokratisierung politischer Ordnung jenseits des Nationalstaats ergeben.

Die Annahme, dass Demokratie eine »wirksame Beteiligung von >unten « 18 verspricht, setzt ein individualistisches Gesellschaftsmodell voraus, in welchem der Staat »oben " und die Individuen »unten « vorgestellt werden. Doch im Bewusstseinshorizont unserer Zeit kann die aktive politische Beteiligung der Bürger allein die Legitimität politischer Ordnung nicht begründen. Es ist nämlich nicht irgendeine Partizipation, sondern erst die gleichberechtigte Beteiligung an dem, was »oben « passiert, die die politische Ordnung rechtfertigt. Die Aussicht auf gleiche individuelle Einflussnahme auf das Zustandekommen politischer Entscheidungen macht das Versprechen der Demokratie aus.

Im Lichte dieser Transformation des politischen Denkens sieht die Habermas'sche deliberative Demokratietheorie in der modernen Verbindung der Begriffe Demokratie und Repräsentation mehr als eine bloße Geburt pragmatischer Notwendigkeit. Im Einklang mit der Interpretation des Repräsentationsbegriffs durch Nadia Urbinati und Mark Warren nimmt sie an, dass eine auf Wahlen basierende repräsentative Demokratie uno actu beides vollzieht: Sie konstituiert die Perspektive einer politischen Gemeinschaft, und sie realisiert den Wert gleicher individueller Freiheitsrechte in der politischen Praxis. »Wenn wir wählen, machen wir zwei Dinge auf einmal: Wir tragen zur Bildung einer Regierung oder Opposition bei, und wir streben die Repräsentation unserer Meinungen und Präferenzen an. Das bedeutet, dass Wahlen nicht einfach einem Wettlauf gleichen, in dem manche auf Kosten anderer gewinnen, sondern eine Teilnahme an der Erschaffung eines repräsentativen Körpers. " ${ }^{19}$ In dieser Lesart bedeutet die moderne Verbindung von Demokratie und Repräsentation keine Abschwächung, sondern einen Bedeutungswandel der parti-

16 Vgl. Skinner 1989.

17 Siedentop 1983, S. 58; Übersetzung D.G.

18 Buchstein, Jörke 2003, S. 482.

19 Urbinati, Warren 2008, S. 398; Übersetzung D.G. Für alternative Interpretationen des Begriffs demokratischer Repräsentation vgl. Pitkin 1967; Schumpeter 1946; Sartori 1965. 
zipativen Komponente des Demokratiebegriffs. Sie vereint zwei spannungsreiche Bedeutungen von politischer Partizipation, indem sie die Institutionalisierung einer Gemeinschaftsperspektive mit der Institutionalisierung der Möglichkeit individueller politischer Selbstbestimmung verbindet - und damit erst den Begriff von Demokratie mit dem Versprechen unterlegt, die für alle gleiche individuelle Freiheit im Rahmen der gemeinsamen politischen Ordnung zu realisieren.

Wenn aber das Versprechen der Demokratie in diesem Sinne als gleichberechtigte individuelle Beteiligung am Zustandekommen politischer Entscheidungen verstanden wird, verliert die Gegenüberstellung der Qualität der Entscheidungen und Partizipation als voneinander unabhängige Aspekte demokratischer Legitimation in einer wichtigen Hinsicht an Plausibilität. Die Vorstellung von gleicher Beteiligung als einem allein auf den »Input « individueller Willens- und Meinungsäußerungen gerichteten Verfahrenskriterium überzeugt nicht, weil mit ihr unweigerlich ein bestimmtes Qualitätsmerkmal des Verfahrensergebnisses impliziert ist: In den demokratischen Entscheidungen muss sich nämlich der gleiche Einfluss aller über Partizipation eingespeisten Stellungnahmen auch zum Ausdruck bringen. Nur dann können die Entscheidungen als gemeinsame Entscheidungen aufgefasst werden. In diesem Sinn kann eine Demokratietheorie nicht umhin, mit ihren Legitimitätskriterien zugleich bestimmte Annahmen über die epistemische Qualität der Entscheidungen zu verbinden, welche durch die Verarbeitung dessen, was in das Entscheidungsverfahren eingespeist wird, erzeugt wird. Die Unterscheidung von Konzeptionen input- und outputorientierter demokratischer Legitimation ${ }^{20}$ ist zumindest in dieser Hinsicht ebensowenig trennscharf wie der pauschale Einwand gegenüber der deliberativen Demokratietheorie, sie würde ihre Legitimitätskriterien an der epistemischen Qualität der Ergebnisse ausrichten. Das Versprechen der Demokratie, welches sich mit der semantischen Transformation zum modernen Demokratiebegriff im Alltagsverständnis etabliert hat, ist letztlich ein Versprechen auf eine rationale Erzeugung kollektiv verbindlicher Entscheidungen in einem spezifischen Sinn: Entscheidungsunterworfene müssen einen Grund zur Annahme haben, dass die zustande gekommenen Entscheidungen auf dem gleichen Einfluss der in nichtexklusiver individueller Beteiligung eingespeisten Stellungnahmen beruhen und daher als gemeinsam getroffene politische Entscheidungen gelten können. Diesen epistemischen Sinn von Demokratie in seiner Bedeutung und seinen Konsequenzen nachzuvollziehen ist eine Aufgabe, die im Mittelpunkt der Theorie deliberativer Demokratie steht.

\section{Qualität statt Gleichheit?}

Diesem Argument könnten Vertreter eines voluntaristischen Verständnisses ${ }^{21}$ von Demokratie Folgendes entgegenhalten: Warum sollte der demokratische Prozess

20 Vgl. Scharpf 1999, S. 16-28.

21 Zur Unterscheidung von voluntaristischem und epistemischem Demokratieverständnis vgl. Fußnote 5. 
eine epistemische Funktion haben, wenn er doch gewährleisten soll, dass »Entscheidungen möglichst unverfälscht aus der gleichen Partizipation aller hervorgehen «? ${ }^{22}$ Ein demokratischer Prozess, der dem Bürgerwillen nichts hinzugibt, sondern gewissermaßen ungefiltert in Entscheidungen münden lässt, fügt letzteren keine Qualität hinzu, die nicht bereits über die gleiche Partizipation in das System Eingang gefunden hat. Das Gleichheitsideal ist durch das Verfahren in Bezug auf den Input des demokratischen Prozesses erfüllt, ohne dass ein Qualitätskriterium der Entscheidungen hinzutritt, so der Einwand.

Der Schwachpunkt dieser Sichtweise lässt sich mit einem Argument aufzeigen, dass von Gary Schaal und Felix Heidenreich vorgetragen worden ist. Schaal und Heidenreich gehen davon aus, dass sich in der modernen, liberalen Demokratie der Begriff politischer Gleichheit aus zwei Komponenten, prozeduraler und substanzieller politischer Gleichheit, zusammensetzt. ${ }^{23}$ Im Rückgriff auf Dahl ${ }^{24}$ erläutern die Autoren Demokratie als das beste aller Regierungssysteme, weil es die Idee der Gleichheit institutionell am weitreichendsten verwirkliche. Dabei beschränke sich die Forderung der Gleichheit nicht nur auf den prozeduralen Aspekt der gleichberechtigten Artikulation individueller Interessen. Vielmehr schließe sie auch den Anspruch der Bürger mit ein, »dass ihre Interessen sakkurat interpretiert< [Dahl] werden «. ${ }^{25}$ Dieses substanzielle Kriterium der Gleichheit im Prozess der Entscheidungsfindung ergebe sich, wie die Autoren unter Bezug auf Saward ${ }^{26}$ herleiten, wesentlich aus dem Umstand einer unüberwindlichen Fallibilität in der Politik. Weil es in der politischen Praxis kein Expertenwissen gebe, das zu »richtigen « oder »wahren « Entscheidungen führe, »ist jede Präferenz der BürgerInnen gleichgewichtig neben jede andere zu stellen $" .{ }^{27}$ Neben der prozeduralen Gleichheit einer gleichberechtigten Artikulation müsse Demokratie also die substanzielle Gleichheit einer gleichgewichteten Einflussnahme der artikulierten Sichtweisen auf das Zustandekommen der Ergebnisse sichern.

Nun bringen Schaal und Heidenreich das Argument der zweidimensionalen politischen Gleichheit aber nicht vor, um eine Schwäche des voluntaristischen Demokratieverständnisses aufzuzeigen, sondern um eine Kritik an der Theorie deliberativer Demokratie vorzubereiten: »Unsere These lautet, dass die deliberative Demokratietheorie nur das Ideal prozeduraler politischer Gleichheit vertritt, jenes der substanziellen politischen Gleichheit jedoch verletzen muss «. ${ }^{28} \mathrm{Um}$ das Ideal politischer Gleichheit zu erfüllen, dürfen Schaal und Heidenreich zufolge keine artikulierten Interessen in der Entscheidungsfindung übergangen werden, und das, so die Autoren weiter, erreiche man, indem »die Präferenzen der BürgerInnen und nur

22 Scharpf 1970, S. 25.

23 Schaal, Heidenreich 2007.

24 Dahl 1989.

25 Schaal, Heidenreich 2007, S. 25.

26 Saward 1994.

27 Schaal, Heidenreich 2007, S. 26.

28 Ebd., S. 24; Hervorhebung im Original. 
die Präferenzen der BürgerInnen vermittelt über freie, gleiche, geheime und allgemeine Wahlen in den politischen Prozess eingespeist werden ${ }^{29}{ }^{29}$ Diese Bedingungen sehen sie im Modell deliberativer Demokratie dadurch verletzt, dass es auf die »Verbesserung der Entscheidungsergebnisse qua Deliberation «30 abstelle. Deliberation wirke demnach wie ein Filter, der auf der Basis eines (je unterschiedlichen) politischen Gütekriteriums bestimmte Präferenzen aussiebe, die zwar artikuliert wären, aber auf die Entscheidung letztlich keinen Einfluss nähmen. Das deliberative Demokratiemodell, so der Schluss der Autoren, sei paternalistisch und undemokratisch, da »nicht alle Beteiligten den gleichen Einfluss auf die endgültige kollektive Entscheidung besitzen «.31

Der Hinweis auf die zwei Dimensionen der Realisierung politischer Gleichheit im demokratischen Prozess ist demokratietheoretisch zweifellos von hoher Relevanz. Fraglich ist jedoch, ob die Theorie deliberativer Demokratie der richtige Adressat dieser Kritik ist. Denn eine wesentliche Motivation des deliberativen Demokratieverständnisses ist es, mit der Hervorhebung der Bedeutung von Deliberation im demokratischen Prozess auf genau jene Gefahr aufmerksam machen zu wollen, die Schaal und Heidenreich als verfehlte Realisierung substanzieller politischer Gleichheit bezeichnen. Gegenwärtige Theorien deliberativer Demokratie sind hervorgegangen aus der Kritik einer ehemals vorherrschenden ökonomischen Deutung liberaler Demokratie, welche Demokratie in Analogie zum Markt als social choice, als organisierten Prozess kollektiver Wahlentscheidungen begreift. In dieser Vorstellung hat der demokratische Prozess vorrangig die Funktion der Aggregation individueller Präferenzen. ${ }^{32}$ Schreibt man jedoch wie ökonomische Ansätze dem demokratischen Prozess primär die Aufgabe zu, eingehende Interessen so zu bündeln, dass zwischen einem Mehrheitswillen und abweichenden Minderheitspositionen unterschieden werden kann, verfehlen demokratische Entscheidungen grundsätzlich das Kriterium substanzieller Gleichheit im Sinne Schaals und Heidenreichs. Statt einer gleichen Einflussnahme aller sind dann in demokratischen Entscheidungen allein die Präferenzen der Mehrheit berücksichtigt. Der demokratische Prozess führt zu der Situation von Gewinnern, denen das Ergebnis der Entscheidung in die Hände spielt, während die Verlierer im Regen stehen - the winner takes it all, the loser's standing small. "Substanzielle« politische Gleichheit besteht dann allenfalls hinsichtlich der gleichen Chance, mit den eigenen artikulierten Interessen die Entscheidung letztlich zu beeinflussen - nämlich dann, wenn es sich ergeben sollte, dass man mit seiner Präferenz (zufällig) auf der Seite der Mehrheit ist. In diesem Sinne werden also gerade im aggregativen Modell von Demokratie bestimmte Präferenzen systematisch herausgefiltert, und zwar alle Präferenzen der Minderheit.

29 Ebd., S. 27.

30 Ebd.

31 Ebd.

32 Die ökonomische Theorie der Demokratie geht zurück auf Schumpeter 1946 und Downs 1968. 
Tatsächlich wird kaum jemand einer solchen Beschreibung des demokratischen Prozesses zustimmen, ohne darauf hinzuweisen, dass im Prozess der Aggregation stets bestimmte Präferenzen der Minderheit berücksichtigt werden müssen. Der entscheidende Punkt ist jedoch, dass man mit dieser Annahme das Parkett der Interessenaggregation als Beschreibung der primären Funktion des demokratischen Prozesses schon verlassen hat. Auf diesen Punkt beziehen sich frühe Kritiken von der Seite deliberativer Demokratietheorie und verweisen auf die Bedeutung von Deliberation in der Demokratie. ${ }^{33}$ Erkennt man die Berücksichtigung von Minderheitsinteressen als Aufgabe der Demokratie an, verliert die Annahme, dass der demokratische Prozess die eingespeisten individuellen Bürgerwillen möglichst mechanisch in seinen Ergebnissen spiegeln müsse, an Plausibilität. Das liegt im Wesentlichen daran, dass Mehrheits- und Minderheitspräferenzen häufig konfligieren. Entsprechend bedeutet die Berücksichtigung von Minderheitsinteressen im Rahmen der Umsetzung einer Mehrheitsentscheidung eine Qualitätsveränderung des Entscheidungsergebnisses, die mit einem Verständnis von Aggregation nicht hinreichend erfasst werden kann. Konfligierende Präferenzen können nicht einfach zusammengefasst werden, sondern bedürfen einer Vermittlung, um möglichst in gleichem Maße Einfluss auf die zu treffende politische Entscheidung zu erhalten. Erkennt man also die Notwendigkeit der Beachtung von Minderheitsinteressen welcher Art auch immer an, unterstellt man dem demokratischen Prozess eine Vermittlungsfunktion und weist ihm damit die Aufgabe zu, alle artikulierten Interessen auf ihre jeweiligen Gründe hin zu prüfen, um ihnen in gleichem Maße (und gegebenenfalls gegen die Interessen der Mehrheit) Berücksichtigung zu verschaffen.

In diesem Sinne beinhaltet in einer Demokratie die Herstellung substanzieller politischer Gleichheit, wie sie von Schaal und Heidenreich beschrieben wird, ein epistemisches Problem. Aus diesem Grund ist die Alternative - hier Qualität der Entscheidungen, dort politische Gleichheit - falsch. Das vom demokratischen Prozess zu lösende epistemische Problem ergibt sich erst aus dem Umstand, dass in den politischen Prozess konfligierende individuelle Interessen und Meinungen eingespeist werden, deren gleichberechtigter Einfluss auf die politische Entscheidung beansprucht wird. ${ }^{34}$ Kurz gesagt, im Kontext einer Demokratie stellt das Ideal der

33 Vgl. insbesondere Bessette 1980; Elster 1986; Miller 1992.

34 Der Maßstab, an dem sich die Qualität demokratischer Entscheidungen bemisst, ergibt sich also aus der normativen Bedeutung des demokratischen Verfahrens selbst. In diesem Sinn ist der epistemische Sinn, den die Habermas'sche Diskurstheorie dem demokratischen Prozess unterstellt, als »demokratie-intern « zu begreifen. Damit unterscheidet sie sich von alternativen Konzeptionen epistemischer Demokratie, die verfahrensunabhängige Qualitätsstandards für die Legitimität demokratischer Entscheidungen annehmen (vgl. Estlund 2008). Aus der Sicht der Diskurstheorie der Demokratie verfehlen solche Ansätze die Bedingungen demokratischer Politik. 
politischen Gleichheit aller Individuen ein Legitimationskriterium dar, in dem " prozedurale Gleichheit « untrennbar mit »substanzieller Gleichheit « ${ }^{35}$ verbunden ist.

Es fragt sich nun, durch welche Eigenschaften der demokratische Prozess auf Seiten der Entscheidungsunterworfenen die Vermutung begründen kann, die Aufgabe der Vermittlung unterschiedlicher politischer Positionen so gut wie eben möglich zu bewerkstelligen. An dieser Stelle verweist die Theorie deliberativer Demokratie auf die zentrale Bedeutung von Deliberation als Modus demokratischer Verarbeitung der eingespeisten Interessen und Stellungnahmen. Es ist aber wichtig zu beachten, dass nicht irgendeine, sondern allein eine Deliberation unter ganz bestimmten Kontextbedingungen die epistemische Funktion erfüllen kann, die ihr im Rahmen eines demokratischen Prozesses zukommen soll. Ein diskursiver Prozess der Willensbildung und Entscheidungsfindung kann letztlich nur dann überzeugen, wenn am Zustandekommen politischer Entscheidungen allen Entscheidungsunterworfenen in gleichem Maße die Möglichkeit der Beteiligung gegeben war, wenn es sich also um einen nichtexklusiven Prozess der Deliberation handelt. Aus diesem Grunde läuft Schaals und Heidenreichs Einwand, die deliberative Demokratietheorie setze zur "Verbesserung der Entscheidungsergebnisse qua Deliberation " das Herausfiltern bestimmter artikulierter Interessen mittels "Schleusen « voraus und verletze so das Ideal politischer Gleichheit, ${ }^{36}$ ins Leere. Um das besser zu verstehen, soll nun genauer nachgezeichnet werden, welche Qualität politischer Entscheidungen die Theorie deliberativer Demokratie unterstellt (Abschnitt 2.1) und welche Bedeutung dem Begriff der Schleuse im Modell eines institutionalisierten demokratischen Prozesses für die Erzeugung dieser Qualität zukommt (Abschnitt 2.2).

\subsection{Die Qualität politischer Entscheidungen in der Theorie deliberativer Demokratie}

Die Qualitätskriterien, die die Theorie deliberativer Demokratie voraussetzt, beziehen sich nicht auf die Inhalte demokratischer Entscheidungen. Entsprechend ist es nicht die Güte eingespeister Präferenzen oder Interessen im Hinblick auf eine zu regelnde politische Materie, die in einer Demokratie relevant ist. Die Qualitätskriterien für demokratische Entscheidungen beziehen sich vielmehr auf die Rechtfertigungen, die für eine jeweilige Entscheidung im Verlaufe der Entscheidungsfindung im demokratischen Prozess entwickelt werden. An der Qualität der Rechtfertigung dafür, warum diese Entscheidung und nicht eine (von alternativen Interessen favorisierte) andere Entscheidung getroffen worden ist, hängt deren demokratische Legitimität. Im deliberativen Demokratieverständnis werden also gar keine Interessen oder Präferenzen »ausgesiebt «. Im Gegenteil müssen die gleichberechtigt einge-

35 Das schließt nicht aus, dass eine Demokratie darüber hinaus noch anderen outputorientierten Performanzkriterien genügen muss, will sie dauerhaft ihre Legitimität erhalten (vgl. Easton 1965). Mit derartigen Anforderungen an die Qualität ihres outcome (etwa ein Mindestmaß ökonomischen Wohlstands oder an Sicherheit) dürften aber alle Formen politischer Herrschaft konfrontiert sein. Zur Erläuterung der spezifischen Eigenschaften demokratischer Legitimität tragen sie daher nicht bei.

36 Schaal, Heidenreich 2007, S. 27. 
speisten Interessen darüber hinaus auch im Prozess ihrer Verarbeitung zu einer Entscheidung insofern Gleichberechtigung erfahren, als sie jeweils nur unter Angabe von Gründen, also gerechtfertigt, als Grundlage für die zu treffende Entscheidung akzeptiert oder zurückgewiesen werden dürfen.

Doch welcher Qualitätsanforderung unterliegen demokratische Rechtfertigungen, und worin ist sie begründet? Der deliberativen Demokratietheorie zufolge leiten sich die Gütekriterien nicht aus einer wie auch immer zu verstehenden, von der jeweiligen Regelungsmaterie abhängenden "politischen Wahrheit" ab, sondern letztlich aus einem grundlegenden individuellen moralischen » Recht auf Rechtfertigung ${ }^{37}$ Das moralische Recht auf Rechtfertigung basiert auf dem normativen Grundprinzip, dass nur diejenigen Normen gerecht sind, die sich gegenüber den Normunterworfenen mit akzeptablen Gründen rechtfertigen lassen. Die Frage ist also, welche Konsequenzen sich aus dem Recht auf Rechtfertigung für die demokratische Beschlussfassung ergeben. Insbesondere fragt es sich, nach welchen Kriterien sich die Akzeptabilität von Rechtfertigungen im politischen Kontext bemisst. Eine Antwort darauf, so argumentiert Rainer Forst, erschließt sich durch folgenden rekursiven Gedankengang: »Da die zu rechtfertigenden Normen für eine jede Person gleichermaßen verbindlich sein sollen und damit einen Anspruch auf wechselseitige und allgemeine Geltung erheben, müssen die sie legitimierenden Gründe selbst wechselseitig und allgemein akzeptabel sein. Reziprozität bedeutet, dass in politischen Argumentationen niemand ein Recht oder bestimmte Güter fordern darf, die er anderen vorenthält. [...] Allgemeinheit heißt, dass alle, die den Normen unterworfen sein werden, gleiche Chancen haben müssen, ihre Ansprüche und Begründungen vorzubringen; die Rechtfertigungsgemeinschaft muss mit der Geltungsgemeinschaft übereinstimmen « ${ }^{38}$

Demzufolge beziehen sich die Kriterien für die Legitimation politischer Entscheidungen zwar auf die Qualität der Gründe, mit denen diese Entscheidungen gegenüber den Normunterworfenen gerechtfertigt werden können: Im demokratischen Prozess der Entscheidungsfindung müssen Rechtfertigungen generiert werden, die den Kriterien der Reziprozität und Allgemeinheit entsprechen. Beide Kriterien stellen jedoch Bedingungen dar, die bestimmte Eigenschaften der Verfahren, in denen politische Rechtfertigungen erzeugt werden, notwendig voraussetzen. Anders gesagt, die Qualität politischer Entscheidungen ist wesentlich (wenn auch nicht ausschließlich) von den Eigenschaften des demokratischen Verfahrens abhängig, in dem sie hervorgebracht worden sind. Welche Implikationen es sind, die sich aus den

37 Forst 2007. Habermas hat jüngst gegenüber Forst eingewendet, dass die Rede von einem Recht auf Rechtfertigung insbesondere im Hinblick auf die Frage der normativen Begründung von Menschenrechten irreführend sei und es sich genau genommen um eine universale und reziproke moralische Pflicht zur Rechtfertigung handelt (Habermas 2012, S. 294-298). Diese Auseinandersetzung um das Verhältnis von Moral und Recht hat jedoch, soweit ich das sehe, keine Auswirkungen auf die hier behandelten Aspekte der Theorie deliberativer Demokratie. Da sich die Formulierung eines » Rechts auf Rechtfertigung " mittlerweile im Sprachgebrauch der politischen Theorie etabliert hat, halte ich nachfolgend an ihr fest.

38 Forst 2007, S. 249.

Leviathan, 41. Jg., 2/2013 
Kriterien der Reziprozität und Allgemeinheit für die Institutionalisierung des demokratischen Prozesses ergeben, soll gleich diskutiert werden (Abschnitt 2.2).

An dieser Stelle muss jedoch zunächst die Qualität demokratischer Rechtfertigungen noch genauer bestimmt werden. Das Recht auf Rechtfertigung impliziert nämlich nicht, dass alle politischen Fragen über moralische Diskurse zu entscheiden wären. Der Grund dafür ist, wie Habermas anführt, dass ein politisches Problem die Frage nach dem » was sollen wir tun " stets in drei (nur analytisch klar zu unterscheidenden) Hinsichten aufwirft. Neben der moralischen Frage, welches Verhalten angesichts der zu regelnden Materie gerecht wäre, ist auch die ethische Frage aufgeworfen, welches Verhalten im Lichte je » unserer « Tradition und Geschichte angemessen und gewünscht wäre, sowie die pragmatische Frage "was können wir tun «. ${ }^{39}$ Daher wäre eine politische Entscheidung, die nur moralisch gerechtfertigt wäre und die ethischen und pragmatischen Aspekte der Regelungsmaterie unberücksichtigt lässt, inakzeptabel. Auf der anderen Seite haben aber moralische Gesichtspunkte in der Bearbeitung eines politischen Problems eine hervorgehobene Bedeutung. Nicht jede politische Entscheidung, die moralisch vertretbar ist, ist deswegen legitim (weil sie in ethischer und pragmatischer Hinsicht unzureichend sein kann) - aber jede politische Entscheidung, die gegen moralische Grundsätze verstößt, ist illegitim.

Vor diesem Hintergrund sind zwei Aspekte zu beachten, um die Konsequenzen des moralischen Grundrechts auf Rechtfertigung für die Legitimität demokratischer Entscheidungen richtig einzuschätzen. Zum einen ist das moralische Kriterium einer reziproken und allgemeinen Rechtfertigung als negatives Kriterium zu verstehen. Anders gesagt, die Legitimität jeder demokratischen Entscheidung steht unter dem Vorbehalt, dass im Prozess ihrer Hervorbringung keine reziproken und allgemeinen Gründe für ihre Zurückweisung ignoriert worden sind. ${ }^{40}$ Dieses Kriterium allein kann aber die für demokratische Legitimität nötige Qualität politischer Entscheidungen nicht begründen. Letztere müssen in einer weiter gefassten politischen Perspektive rechtfertigbar sein, um darüber hinaus auch in ethischer beziehungsweise pragmatischer Hinsicht "ein authentisches Selbstverständnis der Rechtsgemeinschaft, die faire Berücksichtigung der in ihr verteilten Werte und Interessen sowie die zweckrationale Wahl von Strategien und Mitteln ${ }^{41}$ zum Ausdruck zu bringen. Mehr noch als in moralischen Fragen muss aber in ethischen Diskursen über die je problemangemessene Deutung der Werte der politischen Gemeinschaft wie auch in pragmatischen Diskussionen über die Realisierbarkeit von Lösungen angesichts meist unvereinbarer Sichtweisen die Erfüllung des Kriteriums reziproker und allgemeiner Rechtfertigung unerreichbar bleiben. Im Gegenteil wird die demokratische Erörterung von Gründen für und gegen eine Entscheidung im Regelfall nicht durch Konsens, sondern durch formale Mehrheitsabstimmung entschieden. Ein solcher Mehrheitsentscheid führt auf Seiten der Minderheit aber nicht dazu, die Mehrheits-

39 Habermas 1992, S. 196-207.

40 Forst 2007, S. $261 \mathrm{f}$.

41 Habermas 1992, S. 194. 
entscheidung als » besser «, problemangemessener anzuerkennen. Dass Entscheidungen qua Mehrheitsprinzip dennoch akzeptabel sind, hängt von drei Bedingungen ab: erstens von der Einsicht, dass die Herbeiführung einer Entscheidung unumgänglich ist, obwohl die Erörterung von Gründen nicht zur Überwindung konfligierender Sichtweisen geführt hat; zweitens davon, dass die Entscheidung das Minimalerfordernis erfüllt, als im Einklang mit Grundsätzen der Gerechtigkeit stehend rechtfertigbar zu sein; und drittens von der Erwartung, dass die politische Auseinandersetzung um die Materie in Zukunft wieder aufgenommen werden kann und die Chance auf einen Wechsel der Mehrheitsverhältnisse besteht. Für die Definition der Qualität demokratischer Entscheidungen ergibt sich daraus ein zweiter wichtiger Aspekt. Die Qualität, die der Austausch von Gründen im deliberativen Prozess der Willensbildung und Entscheidungsfindung demokratischen Entscheidungen verleiht, ist auf das Zusammenspiel von zwei unterschiedlichen, normativ aufeinander aufbauenden Ebenen der Rechtfertigung zurückzuführen. Wenn im demokratischen Prozess »Entscheidungen getroffen werden, genügt es, wenn diese auf Gründen beruhen, die (a) nicht moralisch zurückweisbar sind und (b) prinzipiell als allgemein akzeptabel gelten, obwohl sie (c) nicht durchgängig als die besten verfügbaren Gründe anerkannt werden (wobei Streit darüber besteht, welches die besten Gründe sind). Dann ist die Übereinkunft als gerechtfertigt anzusehen, obwohl sie nicht auf geteilten Gründen beruht und nicht von allen als beste Lösung angesehen wird «. ${ }^{42}$

Aus dem hierarchischen Verhältnis zwischen der Ebene strikt moralisch-politischer Rechtfertigung und der Ebene allgemein-politischer Rechtfertigung erklärt sich auch die Annahme deliberativer Demokratietheorie, dass dem demokratischen Prozess eine Dynamik der Selbsttranszendierung innewohnt. Der Umstand, dass die Legitimität einer demokratisch getroffenen Entscheidung immer unterlaufen wird, wenn sie gegen moralische Gründe verstößt, gleicht einer (wenn auch schwachen) praktischen Nötigung dazu, nicht nur bestimmte Interessen der Minderheit zu berücksichtigen, sondern darüber hinaus gegebenenfalls die Perspektive bislang unberücksichtigter, auch jenseits der Grenzen der eigenen politischen Gemeinschaft liegender Interessen und Sichtweisen in die Entscheidungsfindung einzubeziehen. Nur in diesem Sinne unterstellt die Theorie deliberativer Demokratie dem demokratischen Prozess eine immanente Tendenz zur Verbesserung der Qualität seiner Entscheidungen: "Die Demokratie wird nur auf demokratische Weise legitim kritisiert. Es mag bessere Antworten als die geben, die ein demokratischer Prozess erzeugt hat, aber die Bedeutung von >besser ist: wechselseitig und allgemein zu rechtfertigen [...] Deliberative Demokratie ist damit die Institutionalisierung der Selbstkorrektur, und Selbstkorrektur heißt, dass die Autorität, ihre Autorität anzuzweifeln, nur bei den Begründungen der Bürger liegt, die diese einander liefern und schulden. Es gibt keine Herrschaft der Gründe ohne die Selbstbestimmung von Bürgern in Form einer diskursiven Praxis des wechselseitigen - auch agonalen - Begründens $« .43$

42 Forst 2007, S. 251.

43 Ebd., S. 267; Hervorhebung im Original.

Leviathan, 41. Jg., 2/2013 


\subsection{Die Schleusenfunktion im Modell deliberativer Politik}

Das Legitimitätskriterium einer diskursiv erzeugten Rechtfertigung demokratischer Entscheidungen mag zunächst realitätsfern klingen. Tatsächlich entwickelt die Theorie deliberativer Demokratie damit jedoch keine normative Utopie, sondern beansprucht, den normativen Sinn eben jenes institutionalisierten politischen Prozesses zu rekonstruieren, der in nationalstaatlichen Demokratien vorliegt. ${ }^{44}$ Demnach bleiben Interessenaggregationen durch Verhandlung und Mehrheitsentscheidung in nationalstaatlichen Demokratien, obwohl sie numerisch betrachtet den Regelfall politischer Alltagspraxis darstellen, in einen Kontext institutionalisierter politischer Diskurse eingebettet. Normativ betrachtet, stehen sie daher nicht auf eigenen Beinen, sondern dienen lediglich als pragmatisches Mittel zur Herbeiführung von Entscheidungen. Um die diskursive Struktur realer Demokratien nachzuvollziehen, entwickelt die Theorie deliberativer Demokratie eine Modellvorstellung des institutionalisierten politischen Prozesses und greift dazu unter anderem auf das Bild einer Schleuse zurück. Deren primäre Funktion besteht aber, wie nun gezeigt werden soll, nicht in der Exklusion, sondern im Gegenteil in der Inklusion der Pluralität gesellschaftlicher Sichtweisen in den Entscheidungsprozess. Es soll eine größtmögliche Zahl von Stellungnahmen aus der Öffentlichkeit in den institutionalisierten politischen Prozess eingeschleust werden. Das wird freilich nur deutlich, wenn man den Begriff der Schleuse im Zusammenhang des Habermas'schen Modells deliberativer Politik betrachtet. ${ }^{45}$

Nach dem Verständnis der deliberativen Konzeption ist die Rationalität der Ergebnisse eines demokratischen Prozesses von einem komplexen Netz aus beidem - institutionalisierten politischen Verhandlungen sowie vorausgehenden Diskursen - abhängig. Verhandlungen haben den Sinn, über den Ausgleich widerstreitender Interessen Kompromisse herbeizuführen. Normativ betrachtet, sind Verhandlungen in einer Demokratie allerdings an zwei Bedingungen gebunden. Erstens müssen sie als faire Verhandlungen gelten. Das bedeutet, sie müssen unter rechtlich fixierten Verfahrensregeln ablaufen, die auf den Ausgleich von Machtasymmetrien zielen. Zweitens stehen die Ergebnisse jeder Verhandlung, wie schon erläutert, unter dem moralischen Vorbehalt, dass sie nicht gegen reziproke und allgemeine Interessen verstoßen dürfen. Beide Bedingungen setzen aber wiederum die Einrichtung anderer Verfahren, nämlich politischer Diskurse voraus. Anders gesagt, in einer demokratischen Politik ist die Legitimität der Ergebnisse von Verhandlungen vom Kontext institutionalisierter Diskurse abhängig, die ihrerseits gleiche, nichtexklusive Einflussmöglichkeiten für alle Normunterworfenen voraussetzen. Für die Etablierung eines solchen Netzes von Verhandlungen und Diskursen haben nun dem Modell deliberativer Politik zufolge dieselben politischen Institutionen vorrangige Bedeutung: eine auf freien und gleichen Wahlen beruhende parlamentarische Repräsen-

44 Vgl. Gaus 2009.

45 Vgl. zum Folgenden Habermas 1992, Kapitel IV, VII, VIII, insbesondere S. 187-208, 358-374 und 426-435. Habermas stützt sich in der Erläuterung des Modells deliberativer Politik stark auf Peters 1993, Kapitel 9.2. 
tation einerseits sowie ein bestimmtes Verhältnis zwischen einem derart rechtlich institutionalisierten parlamentarischen Prozess und einer nichtorganisierten Öffentlichkeit andererseits.

\section{Die Bedeutung von Repräsentation und Wablen im Modell deliberativer Demokratie}

Zunächst gilt es, den unterschiedlichen Sinn zu erkennen, den ein und dieselbe Institution parlamentarischer Repräsentation im Hinblick auf politische Verhandlungen beziehungsweise Diskurse hat. Die Wahl von Repräsentanten hat im Hinblick auf den gesellschaftlichen Interessenausgleich den Sinn der Delegation eines Mandats zur Aushandlung von Kompromissen. »Der Wahlmodus muß für eine faire Vertretung und Aggregierung von gegebenen Interessenlagen und Präferenzen sorgen. « 46 Sobald es jedoch um ethische oder moralische Aspekte einer Entscheidungsmaterie geht, bricht die Prämisse gegebener Wählerpräferenzen, zwischen denen ein Kompromiss zu finden ist, weg. Die Aufgabe des parlamentarischen Prozesses ist dann nicht mehr der Ausgleich zwischen einer Vielzahl bestehender politischer Willen, sondern die Formierung eines erst zu generierenden gemeinschaftlichen politischen Willens. Damit kommt ein zweiter Sinn der Repräsentation ins Spiel: Die Abgeordneten werden zugleich als Teilnehmer an repräsentativ geführten ethischen und moralischen Diskursen bestimmt. Die Wahl bedeutet in dieser Hinsicht nicht die Delegation von Verhandlungsmacht, sondern hat den Sinn einer möglichst breiten Einbeziehung gesellschaftlicher Sichtweisen in die parlamentarischen Beratungsprozesse. Hier soll »der Wahlmodus [...] eine über Personalentscheidungen vermittelte Inklusion aller jeweils relevanten Deutungsperspektiven sicherstellen « ${ }_{4}{ }^{4}$

Dieser zweifache Sinn parlamentarischer Repräsentation spiegelt gewissermaßen die beiden Bedeutungskomponenten politischer Partizipation, wie sie von Urbinati und Warren erläutert worden sind - die institutionelle Möglichkeit der Realisierung individueller politischer Autonomie einerseits, die Institutionalisierung einer Gemeinschaftsperspektive andererseits. Allerdings dürfen parlamentarische Beratungsprozesse nicht im Sinne von Stellvertreterdiskursen gedeutet werden, die im Namen der politischen Gemeinschaft und damit anstelle von Diskursen geführt werden, in denen alle Normunterworfenen die gleiche Möglichkeit zur Beteiligung haben. Aus Sicht der deliberativen Demokratietheorie können akzeptable Rechtfertigungen, die die Legitimität politischer Entscheidungen begründen, nur aus Diskursen hervorgehen, in denen die gleichberechtigte und nichtexklusive Einflussmöglichkeit der Normunterworfenen gewährleistet ist. Dass Bürger an diesem Punkt in der repräsentativen Organisation des demokratischen Prozesses zwar eine normative Spannung, aber keinen Widerspruch wahrnehmen, liegt Habermas zufolge an dem besonderen Verhältnis von institutionalisierter parlamentarischer

46 Habermas 1992, S. 225.

47 Ebd.

Leviathan, 41. Jg., 2/2013 
Willensbildung einerseits und der unregulierten allgemeinen politischen Öffentlichkeit andererseits.

\section{Die Rolle der Öffentlichkeit im Modell deliberativer Demokratie}

Aus der Sicht der Theorie deliberativer Demokratie ist der institutionalisierte demokratische Prozess lediglich der organisierte Kern einer ansonsten spontanen, nicht regulierbaren politischen Öffentlichkeit. Ohne diese kann jener keine demokratisch legitimen Entscheidungen erzeugen - wie deliberativ auch immer er intern verfahren mag. Der Grund dafür ist, dass ein repräsentativ geführter Diskurs eine bestimmte Funktion, die für ethische und moralische Verständigung essentiell ist, prinzipiell nicht erfüllen kann. Während repräsentativ geführte parlamentarische Beratungsprozesse auf die Bearbeitung von Problemen und das Abwägen alternativer Lösungsvorschläge gerichtet sind, bleiben sie auf eine unregulierte Öffentlichkeit der Bürger angewiesen, weil diese einen »Entdeckungszusammenhang " 48 darstellt, der auf gesamtgesellschaftliche Probleme sowie relevante Informationen erst aufmerksam macht. Daher muss die institutionalisierte Willensbildung »durchlässig, sensibel und aufnahmefähig bleiben für die Anregungen, die Themen und Beiträge, Informationen und Gründe « ${ }^{49}$ die ihr aus der allgemeinen Öffentlichkeit zufließen. Nur unter dieser Bedingung besteht aus Sicht der Bürger Grund zu der Annahme, dass sie gleichmäßige Teilhabe am Zustandekommen der politischen Entscheidungen haben. Erst wenn die Richtung demokratischer Politik aus dem Zufluss von Stellungnahmen aus der Öffentlichkeit bestimmt ist, kann der Eindruck einer "Selbstprogrammierung " der institutionalisierten Organe politischer Entscheidungsfindung vermieden werden.

Vor diesem Hintergrund veranschaulicht die Theorie der deliberativen Demokratie die Funktion des parlamentarischen Prozesses mit dem Bild einer Schleuse. Am Eingang der Schleuse strömen kontinuierlich Stellungnahmen aus der allgemeinen Öffentlichkeit in die parlamentarischen Beratungsprozesse ein. In der Schleusenkammer zielt der Prozess parlamentarischer Deliberation darauf, die eingeströmte Pluralität gesellschaftlicher Deutungsperspektiven unter Abzug sozialer Machtasymmetrien zu einer öffentlich rechtfertigbaren Entscheidung zu integrieren. Hier wirkt der parlamentarische Prozess tatsächlich wie ein Filter, wobei es darauf ankommt, zu erkennen, wo diese Filterwirkung erzeugt wird. Die Filterwirkung soll zur Vermeidung der Dominanz derjenigen Stellungnahmen beitragen, die aufgrund bloßer gesellschaftlicher Machtungleichgewichte im ungeregelten Diskurs der Öffentlichkeit mit der lautesten Stimme vorgetragen werden. Am Ausgang der parlamentarischen Schleuse bilden dann alle auf diese Weise gerechtfertigten politischen Entscheidungen die normative Grundlage, auf welcher die politische Administration zum Vollzug kollektiven Handelns autorisiert wird. In diesem Sinn begreift die Theorie deliberativer Demokratie die primäre Funktion der parlamentarischen Schleuse als

48 Ebd., S. 373.

49 Ebd., S. 224. 
Inklusion gesellschaftlicher Vielfalt in den institutionalisierten Entscheidungsprozess. Sie dient dazu, Entscheidungen auf der Basis der größtmöglichen Pluralität gesellschaftlicher Sichtweisen zu treffen, und übernimmt damit eine für die demokratische Legitimation politischer Ordnung wesentliche Funktion. Von einer derartigen institutionellen Realisierung des epistemischen Sinns demokratischer Willensbildung und damit von der Einrichtung funktional vergleichbarer diskursiver Strukturen »transnationaler Resonanz « ${ }^{50}$ zwischen dem organisierten politischen Prozess und der Öffentlichkeit ist aus der Sicht deliberativer Demokratietheorie auch die Legitimität transnationaler Politik abhängig.

\section{Legitimation durch Deliberation ohne Demokratie?}

Ein dritter Einwand gegen die Theorie der deliberativen Demokratie in der Diskussion über die Legitimität der Europäischen Union (EU) schließt hier an. Marcus Höreth zufolge lässt sich die häufige Bezugnahme auf die Theorie der deliberativen Demokratie in der Diskussion über die Legitimität der EU dadurch erklären, dass sie eine Absenkung der für nationalstaatliche Demokratien üblichen Legitimationsanforderungen impliziere, in deren Folge die europäische Politik als legitim erscheine. Kurz gesagt, ihre Hervorhebung der Bedeutung von Beratungen und Diskussionen für demokratische Legitimität führe zu einer verzerrten Darstellung der Legitimität der EU. Im Kontext der EU-Politik sei die deliberative Demokratietheorie demnach »überangepasst ", weil sie im Fokus auf Expertendeliberation die gravierenden Legitimationsdefizite der EU ausblende, die dadurch jedoch nicht kompensiert werden könnten. Gegen diese Überangepasstheit richtet sich Höreths Kritik, die bei genauer Betrachtung an zwei unterschiedliche Adressaten gerichtet ist.

Den ersten Adressaten bilden diejenigen Positionen, die die Legitimität der EU im Kontext der Europaforschung aus unterschiedlichen disziplinären Perspektiven diskutieren und dabei - je nach Gegenstand - auch Aspekte der Theorie deliberativer Demokratie heranziehen. Während Höreth zufolge im Kontext nationalstaatlicher Demokratie repräsentative und deliberative Legitimationsmechanismen koexistierten, diskutierten Vertreter der Theorie deliberativer Demokratie die "Vorzüge der Deliberation nicht im Kontext einer auf Repräsentation beruhenden freiheitlichdemokratischen Ordnung «. ${ }^{51}$ Prozessen der Deliberation werde eine die transnationale Politik legitimierende Wirkung unterstellt, welche das Fehlen von aus dem Nationalstaat bekannten demokratischen Institutionen im europäischen Kontext kompensieren könne. Bei dieser Idealisierung exekutivlastiger Politik wird nach Höreth übersehen, dass demokratische Legitimität über einen deliberativen Modus der Entscheidungsfindung hinaus grundlegend einer institutionalisierten Verantwortlichkeit der Regierenden gegenüber den Bürgern bedürfe. »In deliberativ-demokratischen Ansätzen zur EU tauchen konsequenterweise das Europäische Parlament

50 Trenz, Eder 2004.

51 Höreth 2009, S. 312.

Leviathan, 41. Jg., 2/2013 
und mit ihm verbunden eine fundierte Debatte um allgemeine, freie und gleiche Wahlen sowie Reflexionen zur demokratischen Rückkopplung europäischer Herrschaftsausübung mit einem souveränen Wählerwillen kaum mehr auf. «52 Während Demokratie also auf einer institutionalisierten Kopplung zwischen Regierenden und Bürgern beruhe, komme der Fokus auf den Modus der Deliberation Höreth zufolge einer Entkopplung gleich.

Im Hinblick auf die europawissenschaftliche Literatur zur Legitimität des Regierens jenseits des Nationalstaats ist Höreths Kritik durchaus berechtigt. Tatsächlich lässt sich dort die Tendenz feststellen, den Begriff der Deliberation aus seinem demokratietheoretischen Zusammenhang zu lösen und als Mittelpunkt der Suche nach neuen Wegen der Legitimation transnationaler politischer Ordnung überzustrapazieren. Allerdings ist auf die Folgen, die sich mit einer derart selektiven Bezugnahme auf den Deliberationsbegriff verbinden, bereits ausführlich hingewiesen worden. ${ }^{53}$ Eine so verstandene Deliberation ohne Demokratie kann die Legitimationsfunktion eines demokratischen Willensbildungs- und Entscheidungsprozesses weder im Kontext der EU noch im Kontext globaler Politik auch nur annähernd adäquat ersetzen. ${ }^{54}$

Höreths Kritik der Überangepasstheit geht aber über den Vorwurf einer selektiven Bezugnahme auf die Theorie deliberativer Demokratie im EU-Kontext hinaus und richtet sich direkt an das Habermas'sche Modell deliberativer Politik als einen zweiten Adressaten. Hier tritt jene Überverallgemeinerung deutlich hervor, die - wie eingangs erwähnt - für die gegenwärtige Diskurskonstellation um postnationale Demokratie kennzeichnend ist: Die Kritik an der Rezeption der Theorie deliberativer Demokratie in der Europaforschung wird unterschiedslos auf eine Kritik an der Theorie selbst ausgedehnt. Aus der Sicht von Höreth hat Habermas der Perspektive einer Deliberation ohne Demokratie Vorschub geleistet. Demnach sei durch den Hinweis auf die epistemische Funktion demokratischer Willensbildung verdrängt worden, dass »in einer funktionsfähigen verfassungsstaatlichen Demokratie [...] repräsentativ-demokratische und deliberative Momente " 55 koexistierten. Dass dieser Vorwurf zu kurz greift, ist bereits im vorigen Abschnitt erläutert worden. Nach der Habermas'schen Demokratietheorie wird die besagte epistemische Funktion der Demokratie nur durch den Zusammenhang von institutionalisiertem Entscheidungsprozess und politischer Öffentlichkeit erfüllt, welcher wiederum erst durch einen repräsentativen parlamentarischen Prozesses hergestellt wird. An dieser Stelle geht es nun darum, darzulegen, dass aus dem gleichen Zusammenhang auch normative Anforderungen an die Organisation transnationaler Politik erwachsen, die den Rahmen wie auch immer gestalteter Expertendeliberation sprengen. Die Theorie der deliberativen Demokratie hat weitreichende Überlegungen dazu entwickelt, welche institutionellen Bedingungen vorliegen müssten, damit der epistemische Sinn

52 Ebd., S. 315.

53 Vgl. Schmalz-Bruns 1999; Schmalz-Bruns 2005.

54 Vgl. Niesen 2008.

55 Höreth 2009, S. 312. 
moderner Demokratie auch im Kontext transnationaler Politik zur Geltung gebracht werden kann. Das soll nun abschließend an Habermas' jüngsten Überlegungen zur Europäischen Union illustriert werden.

Habermas stellt seine Überlegungen zur Legitimierung transnationaler Politik in den Kontext der Gegenwartsdiagnose einer » postnationalen Konstellation «. ${ }^{56}$ Während demnach einerseits Globalisierung und Pluralisierung zu einem zügellosen gesellschaftlichen Komplexitätswachstum führen, das den politischen Handlungsspielraum der Nationalstaaten beständig einschränkt, ist andererseits die Fähigkeit zur organisierten kollektiven Selbsteinwirkung eine wesentliche Bedingung der Demokratie. Für demokratische Gesellschaften ergibt sich demzufolge »die Forderung, die politischen Handlungsfähigkeiten über nationale Grenzen hinaus zu erweitern, aus dem normativen Sinn der Demokratie selbst «. ${ }^{57}$ Soll aber die Verrechtlichung transnationaler Beziehungen nicht zu einem für Demokratien inakzeptablen Verlust politischer Legitimität führen, darf sie nicht im intergouvernementalen Politikmodus verharren. In dieser Hinsicht steht Habermas zufolge der europäische Integrationsprozess gegenwärtig am Scheideweg "zwischen transnationaler Demokratie und postdemokratischem Exekutivföderalismus «. ${ }^{58}$ Welche Richtung die EU an diesem Punkt einschlagen sollte, ist für Habermas klar: Trotz aller Schwierigkeiten, die sich aus einer erweiterten Grundgesamtheit europäischer Bürger ergibt, »darf sich eine Politik, die den globalisierten Märkten nachwächst, nur in institutionellen Formen vollziehen, die nicht hinter die Legitimitätsbedingungen demokratischer Selbstbestimmung zurückfallen " ${ }^{59}$ Entgegen Höreths Annahme sind aus Habermas' Sicht im Kontext europäischer Politik keine Abstriche in puncto Legitimationserfordernisse zulässig: Der europäische politische Prozess muss das Prinzip der Volkssouveränität zur Geltung bringen, und das heißt, seine Verfahren dürfen das Zusammenwirken von »Deliberation und Inklusion nicht beeinträchtigen « ${ }^{60}$ Demnach gibt es aus Sicht der deliberativen Demokratietheorie zur modernen Form der demokratischen Legitimition, die auf der Verbindung allgemeiner Wahlen und öffentlicher Diskurse beruht, auch in der postnationalen Konstellation keine adäquate Alternative. Weder lassen sich die Legitimationsanforderungen an supranationale politische Ordnung ermäßigen, noch lässt sich demokratische Legitimation »durch eines ihrer Momente (wie Verantwortung, deliberative Rechtfertigung, Transparenz oder Rechtsstaatlichkeit) ersetzen «. ${ }^{61}$

Wie im nationalen Kontext geht es also auch auf europäischer Ebene darum, dem epistemischen Sinn der Demokratie gerecht zu werden, indem die politische Entscheidungsfindung an einen nichtexklusiven Diskurs aller Entscheidungsunterworfenen rückgebunden wird. Die Besonderheit des europäischen Kontextes besteht nun nicht

56 Habermas 1998.

57 Habermas 2011, S. 50.

58 Ebd., S. 48.

59 Habermas 1998, S. 128.

60 Habermas 2011, S. 54.

61 Ebd.

Leviathan, 41. Jg., 2/2013 
darin, dass diese Aufgabe von anderen Typen politischer Institutionen zu erfüllen wäre. Habermas zufolge bedarf es auch auf EU-Ebene desjenigen institutionellen Zusammenhangs, der den demokratischen Prozess im Nationalstaat wirksam institutionalisiert. Es führt kein Weg an auf freien und gleichen Wahlen beruhender parlamentarischer Repräsentation vorbei. Die Inklusionsfunktion der Beratungsprozesse eines gewählten Parlaments, über welche die Stellungnahmen aus der allgemeinen Öffentlichkeit in den politischen Entscheidungsprozess eingeschleust werden, ist für Habermas nicht nur im Kontext europäischer Politik, sondern auch im Kontext globaler Politik unerlässlich. ${ }^{62}$ Für die Theorie deliberativer Demokratie ist dementsprechend deliberative Politik in der EU nicht mit Deliberationsprozessen zu verwechseln, die als Quelle von "good policy performance «63 oder supranationalem Kollisionsrecht angesehen werden, welches Konflikte zwischen den Rechtsordnungen der Mitgliedstaaten reguliert. ${ }^{64}$ Derartige argumentative Austauschprozesse zwischen Exekutiven beziehungsweise Experten können zweifellos wünschenswerte Ergebnisse hervorbringen, aus demokratietheoretischer Sicht bleiben sie aber unzureichend. Elitendeliberation kann nur dann als Bestandteil einer legitimen Politik betrachtet werden, wenn sie in einem institutionellen Kontext eingebettet stattfindet, der sie als lediglich organisiertes Zentrum der Selbstverständigung aller Bürger einer politischen Gemeinschaft begreifbar macht. Dafür muss sie gegenüber einer gesamteuropäischen parlamentarischen Gesetzgebung verantwortlich sein. Nur letztere kann eine hinreichende Durchlässigkeit des institutionalisierten Entscheidungsprozesses für die Stellungnahmen einer europäischen Öffentlichkeit gewährleisten, die freilich, so Habermas, über eine Europäisierung von Interessenverbänden, Parteien und medialer Berichterstattung ${ }^{65}$ erst noch zu erwachsen hätte. ${ }^{66}$

Im Hinblick auf die institutionellen Anforderungen an die Einrichtung einer diskursiven Struktur demokratischer Willensbildung besteht also eine Parallele zwischen nationalem und europäischem Kontext. Das darf jedoch nicht über einen wesentlichen Unterschied hinwegtäuschen, der verhindert, dass eine europäische Demokratie eine bloße Kopie nationalstaatlicher Demokratie sein kann. Beide Projekte unterscheiden sich Habermas zufolge durch ihre jeweilige historische Ausgangslage, die den normativen Sinn der jeweils zu konstituierenden demokratischen Ordnung verändert. Während es sich bei den Gründungen demokratischer Nationalstaaten um eine erkämpfte Demokratisierung bestehender Staatsgewalten handelte, hat der europäische Einigungsprozess den Charakter einer Integration bereits existierender Demokratien. Für Habermas besteht deshalb insofern ein zentraler Unterschied, als die Begründung einer demokratischen transnationalen EU nicht mehr von der Prämisse einer pouvoir constituante ausgehen kann, die aus einer von nationaler Identität zusammengehaltenen Gemeinschaft von Individuen besteht. In

62 Habermas 2009, S. 418.

63 Neyer 2004.

64 Vgl. Joerges 2006.

65 Habermas 2008, S. 106-108.

66 Vgl. aber Eder 2007. 
dem Projekt der Begründung einer europäischen Demokratie begegnen sich die einzelnen Bürger stattdessen in einer doppelten Rolle: als Bürger, die ihr Zusammenleben mit dem Mittel des Rechts in einer gesamteuropäischen Ordnung demokratisch organisieren wollen, und gleichzeitig als Bürger, die bereits mit einer (jeweils nationalen) Gruppe ihr Zusammenleben in einer freiheitlich-demokratischen Ordnung eingerichtet haben, welche jeweils bewahrt werden soll. Während sich für die Mitglieder nationalstaatlicher Demokratien aus der postnationalen Konstellation also einerseits ein normatives Erfordernis ergibt, verloren gegangene Handlungsfähigkeit durch europäische Integration zurückzugewinnen, kann das andererseits nur unter zwei Bedingungen geschehen. Erstens muss es sich um eine Integration in eine demokratische EU handeln, und zweitens müssen dabei die demokratischen Mitgliedstaaten als gleichberechtigte politische Einheiten erhalten bleiben, weil es sich bei ihnen - jenseits jedweden Interesses an der Bewahrung kultureller Eigenheiten - um »bleibende Errungenschaften und lebendige Gestalten einer sexistierenden Gerechtigkeit $<($ Hegel $) \ll 67$ handelt. Für diese Doppelstruktur eines Modells europäischer Demokratie finden sich Habermas zufolge in zwei Entwicklungen der europäischen Integration entgegenkommende empirische Tendenzen. Zum einen spiegelt sich die Teilung der konstituierenden Gewalt in der Bezugnahme auf beide Subjekte - die Staaten beziehungsweise Völker Europas einerseits, sowie die Bürgerinnen und Bürger andererseits -, welche mit dem Vertrag von Lissabon in die europäischen Verträge aufgenommen wurde. Zum anderen hat sich de facto ein Vorrang des europäischen vor dem nationalen Recht etabliert, der nicht durch eine entsprechende europäische Zwangsgewalt gestützt wird. Stattdessen lassen sich die nationalstaatlichen Verwaltungen zum Zwecke der Implementierung europäisch gesetzten Rechts einspannen und erfüllen so zusammengenommen die Rolle einer dezentral organisierten Staatsgewalt, die auf der EU-Ebene fehlt. Für Habermas ist die fehlende zentrale europäische Staatsgewalt kein Hinderungsgrund, sondern im Gegenteil eine normative Bestandsvoraussetzung europäischer Demokratie. Die Beibehaltung einer teilweisen Autonomie der Mitgliedstaaten ist aus der Sicht ihrer Bürger normativ wünschenswert, »weil diese autonomen Einheiten als Garanten der gleichen Freiheit der Staatsbürger noch gebraucht würden «. ${ }^{68}$

Aus den erläuterten institutionellen Bedingungen einer diskursiv strukturierten und nichtexklusiven demokratischen Willensbildung sowie der Denkfigur einer " ursprünglich geteilten Volkssouveränität «, ${ }^{69}$ die aus der besonderen historischen Konstellation europäischer Integration hervorgeht, kritisiert Habermas schließlich den Status quo der europäischen Integration. Während demnach die dezentrale Organisation der Handlungsfähigkeit der EU bereits vorliegt, verfehlt der europäische Gesetzgebungsprozess das Kriterium deliberativ-demokratischer Willensbildung bei weitem. Für die Einrichtung eines entsprechenden demokratischen Prozesses bedürfte es nicht nur der Transnationalisierung der Wahlen zum Europäischen

67 Habermas 2011, S. 72.

68 Ebd., S. 73.

69 Ebd., S. 70.

Leviathan, 41. Jg., 2/2013 
Parlament durch ein einheitliches Wahlrecht und einer stärkeren Europäisierung des Parteiensystems, um dieses zu einem tatsächlich europäischen Parlament zu machen..$^{70}$ Notwendig wären darüber hinaus im Sinne der geteilten pouvoir constituante zwei weitere Vorkehrungen, die eine europäische Demokratie von einem demokratischen Bundesstaat unterschieden. Zum einen müsste der Gesetzgebungsprozess so umgestellt werden, dass »auf allen Politikfeldern [...] zwischen Rat und Parlament ein Gleichgewicht der Kompetenzen hergestellt «71 ist. Zum anderen müsste die Kommission »von Parlament und Rat in symmetrischer Weise abhängen und beiden Institutionen gegenüber verantwortlich sein «. ${ }^{72}$ Schließlich, so fährt Habermas fort, bliebe in einer demokratischen EU für den Europäischen Rat der Staats- und Regierungschefs als » extrakonstitutionelle Macht « intergouvernementaler Herrschaft kein Platz. Erst unter diesen Bedingungen wäre aus der Sicht deliberativer Demokratietheorie mit einer legitimen deliberativen Politik in der EU zu rechnen.

\section{Schluss}

Aus Sicht der Habermas'schen Theorie deliberativer Demokratie ist der epistemische Sinn demokratischer Willensbildung für die Legitimität politischer Ordnung diesseits und jenseits der Grenzen des Nationalstaats entscheidend. Es wurde gezeigt, dass die Theorie deliberativer Demokratie die epistemische Qualität demokratischer Entscheidungen weder an Gütekriterien bemisst, die dem demokratischen Willensbildungsprozess äußerlich sind, noch Abstriche an dem Erfordernis der Institutionalisierung einer nichtexklusiven, für alle normunterworfenen Bürger zugänglichen politischen Willensbildung zulässt.

Die Einwände einer ungebührlichen Orientierung auf die Rationalität demokratischer Entscheidungen und der damit verbundenen Verfehlung des Ideals politischer Gleichheit durch eine Filterwirkung des demokratischen Prozesses sowie der Vorwurf eines überangepassten Fokus auf Elitendeliberation haben ihre Berechtigung gegenüber einer meist selektiven Rezeption deliberativer Demokratietheorie im Kontext der Diskussion über die Legitimität transnationaler Politik. Das demokratietheoretisch hergeleitete Modell deliberativer Politik treffen sie jedoch insofern nicht, als dieses die in den Einwänden angesprochenen Aspekte von Partizipation, Gleichheit und repräsentativer Institutionen nicht vernachlässigt, sondern zusammengenommen als Voraussetzungen der Erfüllung des epistemischen Sinns von Demokratie begreift.

Mit der vorgetragenen Argumentation verbindet sich die Hoffnung, zu einer Rückbesinnung auf den demokratietheoretischen Entstehungszusammenhang der Theorie deliberativer Demokratie beizutragen und sich dadurch der normativen Zusammenhänge zu vergewissern, die sie auch im Kontext des Regierens jenseits

70 Ebd., S. 73.

71 Ebd.

72 Ebd. 
des Nationalstaats für wirksam erachtet: Wie auch immer die durch politische Macht gestützte administrative Regulierung transnationaler Beziehungen organisiert sein mag, ihre Legitimität hängt davon ab, dass sie auf der Basis von demokratisch erzeugtem Recht ausgeübt wird. Letzteres, das ist eine Kernaussage der Theorie deliberativer Demokratie, gilt unbesehen der Komplexität politischer Regelungszusammenhänge. Auf der normativen Grundlage eines individuellen Rechts auf Rechtfertigung ist demokratische Willensbildung an das Zusammenspiel von Deliberation und Inklusion gebunden, welches die Instiutionalisierung einer Reflexionsinstanz voraussetzt, in der die Allgemeinperspektive der politischen Gemeinschaft wie die jeweils individuellen Perspektiven ihrer Mitglieder gleichberechtigt zur Geltung kommen und miteinander vermittelt werden können. Dieser kritische demokratische Maßstab lässt an der Legitimität derzeit bestehender Formen transnationaler politischer Ordnung zweifeln. Der Habermas'schen Diskurstheorie der Demokratie zufolge wirkt er jedoch zugleich als Motor einer Dynamik der Selbstkritik, die moderne nationalstaatlich institutionalisierte Demokratien seit ihrer Geburtsstunde - mit historischen Rückschlägen und Erfolgen - antreibt.

\section{Literatur}

Bächtiger, André; Steiner, Jürg. Hrsg. 2005. Empirical approaches to deliberative democracy. Acta Politica, Special Issue 40, 2+3. Houndmills: Palgrave Macmillan.

Bessette, Joseph M. 1980. »Deliberative democracy: the majority principle in republican government ", in How democratic is the constitution?, hrsg. v. Goldwin, Robert A.; Schambra, William A., S. 102-116. Washington D.C.: AEI Press.

Besson, Samantha; Martí, José L. Hrsg. 2006. Deliberative democracy and its discontents. Aldershot: Ashgate.

Bohman, James; Rehg, William. Hrsg. 1997. Deliberative democracy: essays on reason and politics. Cambridge: MIT Press.

Buchstein, Hubertus; Jörke, Dirk 2003. »Das Unbehagen an der Demokratietheorie ", in Leviathan 31, 4, S. 470-495.

Buchstein, Hubertus 2009. »Bausteine für eine aleatorische Demokratietheorie «, in Leviathan 37, 3, S. 327-352.

Chambers, Simone 2003. "Deliberative democratic theory «, in Annual Review of Political Science 6, S. 307-326.

Cohen, Joshua 1989. »Deliberative democracy and democratic legitimacy «, in The good polity. Normative analysis of the state, hrsg. v. Hamlin, Alan; Pettit, Philip, S.17-34. Oxford: Blackwell.

Dahl, Robert A. 1971. Polyarchy: participation and opposition. New Haven: Yale University Press.

Dahl, Robert A. 1989. Democracy and its critics. New Haven: Yale University Press.

Downs, Anthony 1968. Ökonomische Theorie der Demokratie. Tübingen: Mohr Siebeck.

Dryzek, John 2000. Deliberative democracy and beyond: liberals, critics, contestations. Oxford: Oxford University Press.

Dunn, John 2005. Setting the people free: the story of democracy. London: Atlantic Books.

Easton, David 1965. A systems analysis of political life. New York: Wiley \& Sons.

Eder, Klaus 2007. »Europa als besonderer Kommunikationsraum «, in Berliner Journal für Soziologie 17, 1, S. 33-50.

Elster, Jon 1986. »The market and the forum: three varieties of political theory «, in Foundations of social choice theory, hrsg. v. Elster, Jon; Hylland, Aanund, S. 103-132. Cambridge: Cambridge University Press.

Elster, Jon. Hrsg. 1998. Deliberative democracy. Cambridge: Cambridge University Press. 
Estlund, David 2008. Democratic authority: a philosophical framework. Princeton: Princeton University Press.

Forst, Rainer 2007. »Herrschaft der Gründe. Drei Modelle deliberativer Demokratie ", in Rainer Forst: Das Recht auf Rechtfertigung. Elemente einer konstruktivistischen Theorie der Gerechtigkeit, S. 224-269. Frankfurt a. M.: Suhrkamp.

Gaus, Daniel 2009. Der Sinn von Demokratie. Die Diskurstheorie der Demokratie und die Debatte über die Legitimität der EU. Frankfurt a. M., New York: Campus.

Gutmann, Amy; Thompson, Dennis F. 1996. Democracy and disagreement. Cambridge: Harvard University Press.

Habermas, Jürgen 1992. Faktizität und Geltung. Beiträge zu einer Diskurstheorie des Rechts und des demokratischen Rechtsstaats. Frankfurt a. M.: Suhrkamp.

Habermas, Jürgen 1998. "Die postnationale Konstellation und die Zukunft der Demokratie «, in Jürgen Habermas: Die postnationale Konstellation, S. 91-169. Frankfurt a. M.: Suhrkamp.

Habermas, Jürgen 2009. »Konstitutionalisierung des Völkerrechts und die Legitimationsprobleme einer verfaßten Weltgesellschaft ", in Politische Theorie. Philosophische Texte, Band 4, hrsg. v. Habermas, Jürgen, S. 402-424. Frankfurt a. M.: Suhrkamp.

Habermas, Jürgen 2011. »Die Krise der Europäischen Union im Lichte einer Konstitutionalisierung des Völkerrechts. Ein Essay zur Verfassung Europas «, in Jürgen Habermas: Zur Verfassung Europas, S. 39-96. Frankfurt a. M.: Suhrkamp.

Habermas, Jürgen 2012. "Rawls' politischer Liberalismus. Replik auf die Wiederaufnahme einer Diskussion ", in Nachmetaphysisches Denken II, hrsg. v. Habermas, Jürgen, S. 277-307. Frankfurt a. M.: Suhrkamp.

Höreth, Marcus 2009. »Überangepasst und realitätsentrückt. Zur Paradoxie der Theorie der deliberativen Demokratie in der EU «, in Zeitschrift für Politikwissenschaft 19, 3, S. 307-330.

Joerges, Christian 2006: »Deliberative political processes revisited: what have we learnt about the legitimacy of supranational decision-making ", in Journal of Common Market Studies 44, 4, S. 779-802.

Jörke, Dirk 2009. »The epistemic turn of critical theory: implications for deliberative politics and policy-making «, in Critical Policy Studies 3, 3-4, S. 440-446.

Knight, Jack; Johnson, James 1994. »Aggregation and deliberation: on the possibility of democratic legitimacy «, in Political Theory 22, 2, S. 277-296.

Manin, Bernard 1987. »On legitimacy and public deliberation «, in Political Theory 15, 3, S. 338-368.

Miller, David 1992. »Deliberative democracy and social choice«, in: Political Studies XL, Special Issue, S. 54-67.

Neyer, Jürgen 2004. »Explaining the unexpected: efficiency and effectiveness in European decision-making ", in Journal of European Public Policy 11, 1, S. 19-38.

Niesen, Peter 2008. „Deliberation ohne Demokratie? Zur Konstruktion von Legitimität jenseits des Nationalstaats ", in Transnationale Verrechtlichung. Nationale Demokratien im Kontext globaler Politik, hrsg. v. Kreide, Regina; Niederberger, Andreas, S. 240-259. Frankfurt a. M., New York: Campus.

Passerin D’Entrèves, Maurizio. Hrsg. 2002. Democracy as public deliberation. Manchester: Manchester University Press.

Pitkin, Hanna F. 1967. The concept of representation. Berkeley: University of California Press.

Peters, Bernhard 1993. Die Integration moderner Gesellschaften. Frankfurt a. M.: Suhrkamp.

Rosanvallon, Pierre 2010. Demokratische Legitimität. Unparteilichkeit, Reflexivität, Nähe. Hamburg: Hamburger Edition.

Sartori, Giovanni 1965. Democratic theory. New York: Praeger.

Saward, Michael 1994. "Democratic theory and indices of democratisation ", in Defining and measuring democracy, hrsg. v. Beetham, David, S. 6-24. London: Sage.

Schaal, Gary S.; Heidenreich, Felix 2007. »Quality versus Equality? Liberale und deliberative Ideale politischer Gleichheit ", in Österreichische Zeitschrift für Politikwissenschaft 36, 1, S. 23-38.

Schaal, Gary S.; Ritzi, Claudia 2009. Empirische Deliberationsforschung. MPIfG Working Paper 09/9. Köln: Max-Planck-Institut für Gesellschaftsforschung.

Scharpf, Fritz W. 1970. Demokratietheorie zwischen Utopie und Anpassung. Konstanz: UVK. 
Scharpf, Fritz W. 1999. Regieren in Europa. Effektiv und demokratisch? Frankfurt a. M.: Campus.

Schmalz-Bruns, Rainer 1999. »Deliberativer Supranationalismus. Demokratisches Regieren jenseits des Nationalstaats ", in Zeitschrift für Internationale Beziehungen 6, 2, S. 185-244.

Schmalz-Bruns, Rainer 2005. »On the political theory of the Euro-polity «, in Making the European polity: reflexive integration in the EU, hrsg. v. Eriksen, Erik O., S. 59-83. Oxford: Polity Press.

Schumpeter, Josef A. 1946. Kapitalismus, Sozialismus und Demokratie. Bern: Francke.

Siedentop, Larry 1983. "Political theory and ideology: the case of the state «, in The nature of political theory, hrsg. v. Miller, David; Siedentop, Larry, S. 53-73. Oxford: Clarendon Press.

Skinner, Quentin 1989. »The state «, in Political innovation and conceptual change, hrsg. v. Ball, Terence; Farr, James; Hanson, Russell L., S. 90-131. Cambridge: Cambridge University Press.

Thompson, Dennis F. 2008. »Deliberative democratic theory and empirical political science «, in Annual Review of Political Science 11, S. 497-520.

Trenz, Hans-Jörg; Eder, Klaus 2004. »The democratizing dynamics of a European public sphere: towards a theory of democratic functionalism «, in European Journal of Social Theory 7, 5, S. 5-25.

Urbinati, Nadia; Warren, Mark E. 2008. »The concept of representation in contemporary democratic theory «, in Annual Review of Political Science 11, 1, S. 387-412. 
Zusammenfassung: Theorien deliberativer Demokratie werden dafür kritisiert, sich auf die Qualität politischer Entscheidungen zulasten von Partizipation und Gleichheit zu konzentrieren. Dieser Einwand von »Deliberation ohne Demokratie « trifft auf die Habermas'sche Diskurstheorie der Demokratie nicht zu. Diese unterstellt einen epistemischen Sinn von Demokratie, wonach öffentliche Deliberation gekoppelt mit inklusiver parlamentarischer Repräsentation eine Bedingung der Herstellung politischer Gleichheit und demokratischer Legitimität darstellt - sowohl in nationalstaatlicher als auch in transnationaler Politik.

Stichworte: Demokratietheorie, demokratische Legitimität, deliberative Demokratie, Habermas, Europäische Union, Partizipation, Gleichheit, Diskurstheorie.

\section{Quality versus participation and equality? Some remarks on the epistemic core of democracy}

Abstract: Theories of deliberative democracy are criticised for stressing output quality at the expense of participation and equality. The objection to »deliberation without democracy « does not apply to Habermas' discourse-theory of democracy, which assumes an epistemic function of democracy, according to which public deliberation based on inclusive parliamentary representation is a precondition for political equality and democratic legitimacy - both in the context of national as well as transnational politics.

Keywords: Democratic theory, democratic legitimacy, deliberative democracy, Habermas, European Union, participation, equality, discourse theory.

Autor

Dr. Daniel Gaus

Käte Hamburger Kolleg »Politische Kulturen der Weltgesellschaft «

Universität Duisburg-Essen

Schifferstr. 196

47059 Duisburg

gaus@gcr21.uni-due.de 